\title{
Construção Colaborativa de Signos Específicos da Língua Brasileira de Sinais para Termos da Subárea de Engenharia de Software
}

\author{
José Augusto Fabris ${ }^{1}$, Soraia Silva Prietch ${ }^{2}$, Kefferson Ricardi ${ }^{1}$ \\ ${ }^{1}$ Curso de Ciência da Computação. Faculdade Anhanguera. Av. Ary Coelho, 829 - \\ Cidade Salmen, 78705-050, Rondonópolis-MT, Brasil \\ ${ }^{2}$ Curso de Sistemas de Informação. Universidade Federal de Mato Grosso. Câmpus de \\ Rondonópolis. Av. dos Estudantes, $\mathrm{n}^{\circ}$ 5055. Bairro Cidade Universitária. 78735-901, \\ Rondonópolis-MT, Brasil \\ joseaugustoo2011@hotmail.com, soraia@ufmt.br, \\ keffersonricardi@gmail.com
}

\begin{abstract}
Deaf students are losing interest in the search for knowledge because of the absence of content, books, vocabularies, dictionaries or glossaries with specific academic jargon in Libras. Therefore, this paper presents related researches highlighting the precariousness of terms referring to Software Engineering contents in Libras. The signs constructed and documented in the technical files were submitted to opinion research with people who are deaf, Libras users with completed higher education or in progress. As a result, it was evident from participants' opinion that it is important to construct signs of the Libras for areas of knowledge that still have few signs, such as Computing area.
\end{abstract}

Resumo. Estudantes surdos estão perdendo o interesse na busca do conhecimento por não ter acesso a livros, vocabulários, dicionários ou glossários com jargões acadêmicos específicos em Libras. Portanto, esse artigo apresenta trabalhos correlatos ressaltando a precariedade de termos referentes a conteúdos de Engenharia de Software em Libras. Os signos construídos, e documentados nas fichas técnicas, foram submetidos à pesquisa de opinião de pessoas surdas usuárias da Libras com ensino superior concluido ou em andamento. Como resultado ficou evidente, pela opinião dos participantes, a importância de construir sinais da Libras para áreas de conhecimento que ainda possuem poucos sinais, como a área de Computação.

\section{Introdução}

O ponto de partida para a realização do presente trabalho foi a experiência e o desejo pessoal do primeiro autor, desse artigo, enquanto estudante surdo usuário da Língua Brasileira de Sinais (Libras) de um curso de graduação de Ciência da Computação. O referido estudante vem conquistando seu espaço, tanto no mercado de trabalho como em sua educação formal, usufruindo de seus direitos como sujeito participativo e crítico na sociedade. Contudo, um dos maiores desafios para sua inclusão plena é a barriera da comunicação. Como um sujeito que percebe o mundo através de experiências visuais, 
torna-se evidente a necessidade de uma comunicação mais aberta em relação às duas Línguas Brasileiras: Português e Libras, em especial, no que se refere aos conteúdos e aos jargões específicos de uma área de conhecimento.

A Libras foi reconhecida no Brasil como segunda língua oficial [Lei ${ }^{\circ}$. 10.436 (2002); Decreto $n^{0} 5.626$ (2005)], apesar de ser utilizada pela comunidade surda muito antes desta data. A Libras é um idioma relativamente novo, que sofre mudanças ao longo do tempo, como qualquer outro idioma, e se torna cada vez mais rico. Como indivíduo, o estudante surdo, vê a necessidade de construção de novos signos linguísticos específicos para garantir aprendizagem significativa, enriquecer $\mathrm{o}$ vocabulário $\mathrm{e}$ proporcionar comunicação clara e aberta entre aluno e professor.

Além disso, no município em que a pesquisa foi realizada, não existem intérpretes da Libras com conhecimento profundo ou formação na área de Computação. Antes das aulas existe uma preparação por parte dos profissionais intérpretes; no entanto, durante as aulas, sinais são gerados para tentar suprir a falta de representações coerentes para os termos usados na língua portuguesa para a área específica. Boscarioli et al. (2015) traz um exemplo dessa situação em que o termo "computação em nuvem" foi utilizado em uma aula e, assim como esse, por falta de conhecimento específico na área, diversos sinais são criados sem a compreensão sólida dos conceitos. Ao refletir sobre o assunto, se em cada sala de aula de cursos de computação diferentes sinais estão sendo gerados isoladamente, então torna-se muito difícil garantir igualdade de oportunidades ao público usuário da Libras, tais como, realizar mobilidade acadêmica, participar do Exame Nacional para Ingresso na Pós-Graduação em Computação (POSCOMP), dentre outras.

Com o objetivo de auxiliar no processo de ensino-aprendizagem, surgiu a necessidade da construção colaborativa de signos linguísticos em Libras para termos técnicos para a disciplina de Engenharia de Software presente em cursos da área de Computação, podendo promover melhorias na comunicação, na interação entre os presentes em sala de aula e na compreensão de conteúdos específicos.

\section{Metodologia de pesquisa}

Os procedimentos metodológicos foram realizados conforme a sequência:

- Buscar e assistir aos vídeos disponíveis na Internet a respeito de dicionários, vocabulários, glossários e relação de termos da área de computação/informática em Libras, com foco de atenção para os conteúdos de Engenharia de Software;

- Pesquisar sobre trabalhos correlatos, ou seja, localizar trabalhos na literatura que tenham realizado pesquisa semelhante a esta proposta;

- Efetuar contatos com pessoas surdas que frequentam ou frequentaram cursos na área de computação/informática e com pessoas surdas que trabalham na área de Tecnologia da Informação (TI), a fim de convidá-los posteriormente para participar como voluntários na pesquisa;

- Definir relação de termos selecionados, e seus significados, a partir de livros usados na disciplina de Engenharia de Software para que sinais da Libras sejam propostos;

- Construir os sinais da Libras para o domínio específico, de acordo com os significados/conceitos dos termos selecionados. A construção dos signos linguísticos 
foi realizada pelo estudante surdo, usuário nativo da Língua Brasileira de Sinais, em colaboração com profissional intérprete da Libras (ambos autores desse artigo);

- Elaborar questionário para efetuar pesquisa de opinião sobre os sinais construídos;

- Aplicar questionário em pesquisa de opinião com participantes voluntários, que sejam pessoas surdas ou com deficiência auditiva, de forma presencial, e que, de alguma forma, tiveram contato com a área de computação/informática.

\section{Trabalhos Correlatos}

Para localizar os trabalhos disponíveis na literatura, uma busca exploratória, no Google Acadêmico, foi realizada utilizando as seguintes palavras-chave combinadas: \{\{Glossário $\}$ OR \{Vocabulário\} OR \{Dicionário\}\} AND \{\{Libras\} OR \{Língua Brasileira de Sinais\}\} OR/AND \{\{Computação\} OR \{Informática\} OR \{Engenharia de Software \}\}, somente em língua portuguesa, sem se preocupar com o ano de publicação. A partir dessa busca, alguns trabalhos foram encontrados, sendo que dentre eles 06 (seis) foram considerados mais próximos ao que desejava-se propor na presente investigação. Os trabalhos são os seguintes, os quais são brevemente descritos na sequência, em ordem cronológica de publicação, destacando os pontos mais interessantes: Faqueti, Grandi, Fantini e Lorenzetti (2005); Oliveira e Stumpf (2013); Souza, Lima e Pádua (2014); Kuhn (2015); Borges, Obara, Leite e Rocha (2015); Brochado, Lacerda e Rocha (2016).

O projeto de Faqueti, Grandi, Fantini e Lorenzetti (2005) informa que a pesquisa se iniciou com entrevistas com alunos surdos do ensino médio para se definir os componentes básicos (levantar requisitos) do InfoLibras. Posteriormente, "Foram criados 320 sinais relacionados com palavras específicas da informática. Os sinais foram criados a partir de uma necessidade enquanto acadêmico surdo do Curso de Ciência da Computação [...]" (ibid., p. 2865).

Oliveira e Stumpf (2013) relatam, em seu artigo, sobre o processo de desenvolvimento do Glossário Letras-Libras online, da Universidade Federal de Santa Catarina (UFSC), para sinais acadêmicos. Conforme as autoras (ibid., p. 2017), "o número de estudantes surdos nas universidades, particulares e públicas tem aumentado no Brasil, notadamente na última década", especialmente devido ao Decreto $\mathrm{n}^{\mathrm{o}}$ $5.626 / 2005$. Portanto, a relevância da construção de sinais para domínios específicos ainda está em sua fase inicial e muito precisa ser realizado a esse respeito. Na equipe de criação do Glossário Letras-Livras, Oliveira e Stump (2013, p. 218) mencionam que "A tradução destes termos técnicos demanda discussão permanente da equipe, pesquisa e criação de neologismos a fim de minimizar a dificuldade dos estudantes na compreensão dos textos acadêmicos". Isso porque a Libras é uma língua dinâmica e porque possui diferenças culturais (regionalismos). Inclusive, a equipe trabalha com grupos de outros estados brasileiros para que essas diferenças sejam representadas e registradas. Para a produção dos sinais para o Glossário Letras-Libras, Oliveira e Stumpf (2013) informam que os conteúdos dos vídeos são padronizados seguindo a mesma estrutura: (1) Soletração da palavra; (2) Sinal a ser utilizado pelos tradutores do curso; (3) Explicação do termo; (4) Exemplos; e, (5) Variações regionais. Contudo, "Caso a equipe não conheça um 'sinal' em Libras que corresponda ao termo em português e, ainda não seja possível apresentar uma proposta de neologismopara o termo, o Glossário tem a seguinte apresentação: *Soletração da palavra; *Explicação do termo; *Exemplos" (ibid., p. 
223). Além disso, Tennant e Brown (1998) apud Oliveira e Stumpf (2013, p. 223-224) mencionam que "[...] uma combinação de sinais pode ser suficiente para representar o conceito até que um sinal mais eficiente seja criado pela comunidade surda".

O trabalho de Souza, Lima e Pádua (2014, p. 76) foi motivado por "Dois problemas [...]: 1) o da escassez do léxico terminológico em Libras nos campos da Ciência, Tecnologia, Cultura e Arte e 2) o do ônus da escassez do léxico que recai sobre o estudante surdo tornando seu acesso e permanência nas esferas acadêmicas superiores extremamente dificultado". Os autores realizaram estudo de caso para a proposta de termos em Libras da área de Desenho Arquitetônico.

Complementando o problema da escassez de sinais em Libras para diversas áreas, Souza, Lima e Pádua (2014, p. 81) questionam "Por que não temos dicionários terminológicos bilíngues bimodais, em Libras? De quais maneiras pode-se criar, recolher e/ou validar sinais em Libras? Por que os sinais criados todos os dias nas salas de aulas do País não são registrados e compartilhados?”. Uma demonstração disso, segundo os autores (ibid.), é a comparação entre a edição revisada e aumentada do Novo DeitLibras - Dicionário Enciclopédico Ilustrado Trilíngue da Língua de Sinais Brasileira, de 2013, que contém 9.828 sinais de Libras, e o Dicionário Houaiss em Língua Portuguesa, que contém em torno de 228.500 verbetes.

Souza, Lima e Pádua (2014) proõem uma ficha léxico-terminográfica, adaptada de Lima (2014, p. 122), a qual abarca diversas informações a respeito da construção de um sinal em Libras de determinada área de conhecimento e serve como modelo para a criação da ficha elaborada para uso nesse trabalho. Souza, Lima e Pádua (2014) recomendam que para a criação de um glossário é preciso reunir um grupo de especialistas, a fim de definir e validar os termos que constarão nesse documento ou aplicação. Nesse processo, os autores mencionam a importância de constar "representantes da comunidade surda, surdos que já tenham conhecimento sobre a disciplina, alunos que estão aprendendo a disciplina, e intérpretes" (ibid., p. 86). Isso mostra a importância de se conduzir a construção de signos seguindo o rigor metodológico e considerando as pessoas certas no processo de construção.

A dissertação de Kuhn (2015) propõe a criação de material didático para o curso de Engenharia de Produção em Libras, usando como referência 10 áreas e 55 subáreas regulamentadas pela Associação Brasileira de Engenharia de Produção (ABEPRO). Sendo assim, o material produzido por Kuhn (2015) conta com o total de 65 novos sinais da Libras para a área específica. Foi criado através do estudo dos conteúdos em livros do domínio de interesse para ter sólido embasamento teórico sobre os termos definidos, da produção de vídeo amador e de desenhos técnicos. Para isso, a autora utilizou os 05 (cinco) parâmetros da Libras para a composição dos sinais e das informações contidos nos desenhos técnicos.

Borges, Obara, Leite e Rocha (2015) propõem, em seu resumo, um "Glossário interativo de Libras para a área de Computação", cuja motivação foi a ampliação do acesso e da permanência da pessoa surda em ambiente educacional. Os autores mencionam que existem materiais didáticos adaptados para surdos, porém ainda não é suficiente; pois, muitos termos de áreas profissionais ainda não possuem representação em Libras. A motivação dos autores reforça o que os demais trabalhos correlatos encontrados na literatura também evidenciaram, que ainda não existem sinais suficientes 
em Libras que abranjam diversas áreas de conhecimento específicas. Borges, Obara, Leite e Rocha (2015) informam que, em levantamento preliminar, reuniram 310 termos técnicos da área de Computação/ Informática, e conta com um avatar 3D animado, chamada Stayce. Não ficou claro se os termos levantados se encontram em Libras ou se são termos em língua portuguesa a serem criados.

O resumo de Brochado, Lacerda e Rocha (2016) relata sobre a proposta de projeto de pesquisa em andamento intitulado como "Desenvolvimento de Software Glossário de Informática com Aplicação de Libras e de Tecnologia de Captura de Movimento 3D”. O projeto conta com a parceria entre a Universidade Estadual do Norte do Paraná (UENP) e a Universidade Tecnológica Federal do Paraná (UTFPR)/ Câmpus de Cornélio Procópio. Uma das etapas desse projeto de pesquisa consistiu na definição dos sinais em Libras, realizada em diferentes meios de pesquisa (sendo 120 verbetes analisados), agregando aqueles sinais já utilizados por surdos atuantes na área. Após a seleção, realizada por estudantes de Engenharia da Computação, a relação de sinais resultante foi analisada por uma professora surda, tradutora/ intérprete da Libras, em que descobriu-se variantes de sinais já utilizados por outros grupos.

A partir da leitura dos trabalhos supracitados é possível verificar que ainda existem poucas pessoas surdas em cursos de ensino superior, por esse motivo sinais devem ser criados por pessoas nativas de sua língua, sendo o caso, pois a língua natural dos surdos brasileiros é a Libras. Dentre os trabalhos selecionados, 03 (três) se referem à Dicionários ou Glossários para a área de Computação/ Informática em Libras [Faqueti, Grandi, Fantini e Lorenzetti (2005); Borges, Obara, Leite e Rocha (2015); Brochado, Lacerda e Rocha (2016)]. No entanto, nenhum dos três recursos propostos foram encontrados na Web para uso ou consulta. Portanto, além de construir sinais novos para conteúdos e jargões específicos, ainda se faz necessária a disponibilização e a divulgação desses sinais, de modo que se tornem acessíveis para um grande número de pessoas surdas interessadas pela área de Computação.

\section{Construção de Signos da Libras para a Subárea da Computação - Engenharia de Software}

\subsection{Preparação para a pesquisa de opinião}

Nesta etapa, foi definida a relação de termos, e seus significados, da subárea da Engenharia de Software para que sinais da Libras sejam propostos, a saber: (1) Engenharia de Software; (2) Processos; (3) Modelo de processo/ Ciclo de vida; (4) Modelos de Processos Prescritivos; (5) Modelo Cascata; (6) Modelo Incremental; (7) Modelo Evolucionário; e, (8) Modelo Espiral. A escolha desses termos ocorreu pela afinidade do estudante surdo (primeiro autor desse artigo) com o assunto durante as aulas que teve na disciplina de Engenharia de Software no Curso de Ciência da Computação. Os termos foram definidos utilizando as seguintes referências: Sommerville (2003); Pressman (2011); Wazlawick (2013); e, Paula Filho (2015).

\subsection{Desenvolvimento}

A etapa de preparação para pesquisa foi subdividida em três atividades: construção dos signos da Libras para a subárea Engenharia de Software, preparação e execução da pesquisa de opinião, as quais são descritas na sequência. 
A construção dos sinais da Libras para o domínio específico, de acordo com os significados dos termos escolhidos foi proveniente da interação aluno-intérprete em sala de aula, e de estudo realizado por intérprete junto com o autor (estudante surdo). Para documentar os sinais construídos, levou-se em consideração o conhecimento e os exemplos extraídos do embasamento teórico e dos trabalhos correlatos. Um modelo de ficha foi elaborado para uso nesse trabalho, tomando como base a ficha léxicoterminográfica de Lima (2014, p. 122) apud Souza, Lima e Pádua (2014, p. 85). Para cada um dos 08 (oito) termos foram construídos 03 (três) sinais em Libras, totalizando em 24 fichas preenchidas. Esses sinais foram filmados e os dados referentes aos sinais foram documentados usando o modelo. No Quadro 1, uma ficha preenchida é apresentada a fim de exemplificar a forma de documentação dos sinais.

Quadro 1. Ficha da Opção 1 de sinal para o termo Engenharia de Software.

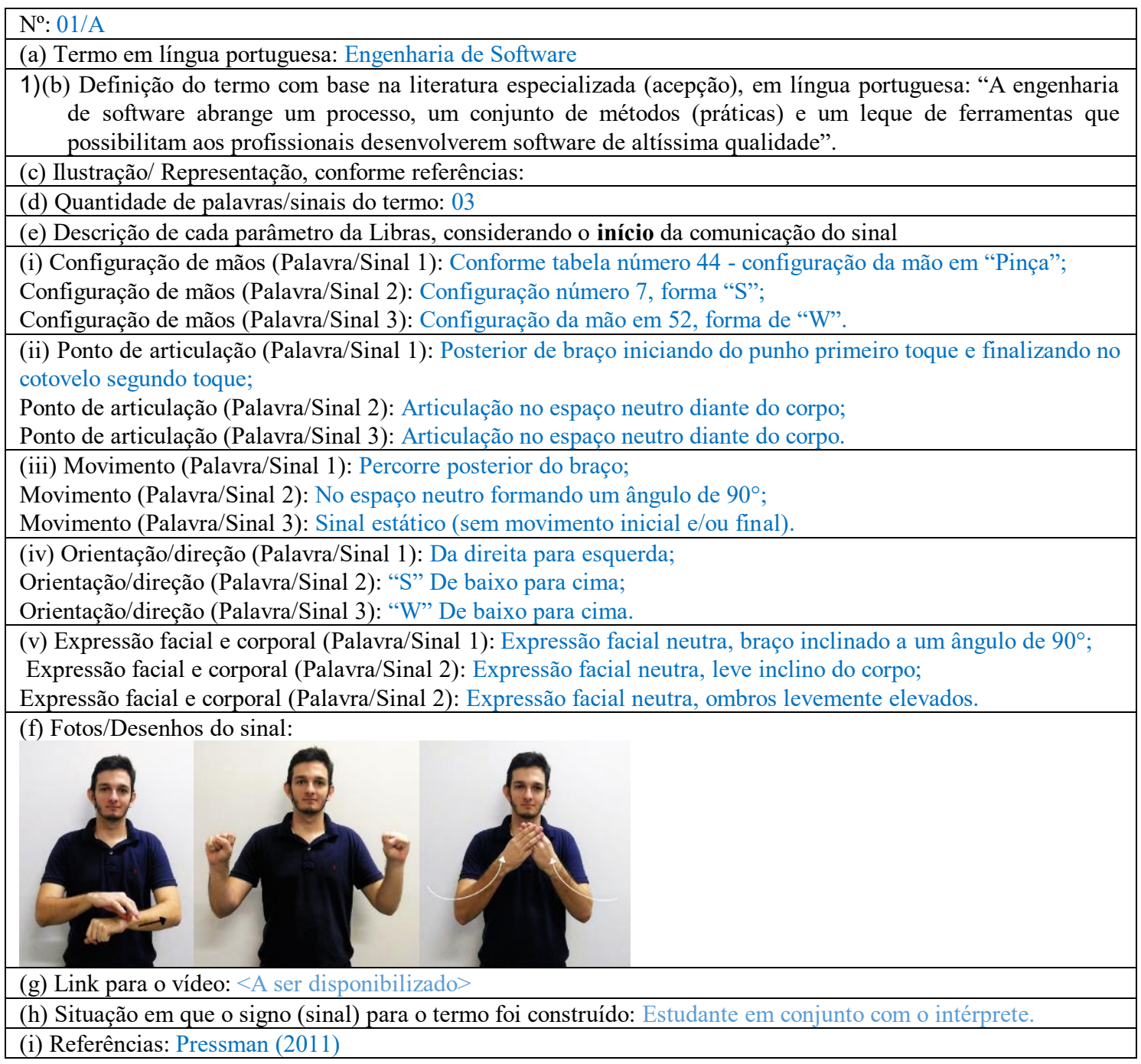

Fonte: Ficha adaptada de Lima (2014, p. 122) apud Souza, Lima e Pádua (2014, p. 85); (Fotos do primeiro autor).

\subsection{Pesquisa de opinião}

Após a elaboração das fichas, iniciou-se a fase de preparação, que consistiu na elaboração de um questionário para verificar se os participantes conheciam sinais em Libras para a relação de termos escolhidos, para identificar o perfil das pessoas surdas 
que conhecem ou que estudaram esses termos previamente e para efetuar votação dentre os sinais construídos. Uma das perguntas do questionário consta ilustrado na Figura 1, com três opções de respostas fechadas (1, 2 ou 3) e uma aberta (4) caso o participante quisesse sugerir um sinal novo ou um sinal aprendido previamente.

\section{Figura 1. Formato de uma das questões do segundo modelo de questionário utilizado na pesquisa de opinião.}

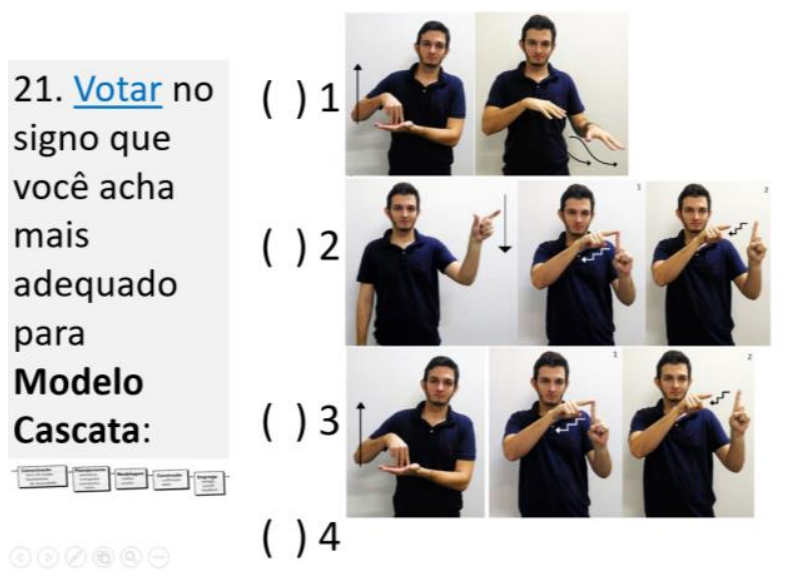

Fonte própria (Fotos do primeiro autor).

Duas pessoas surdas participaram voluntariamente, sendo um egresso do curso de Ciência da Computação e um estudante do curso de Pedagogia. Ambas as participações foram realizadas de forma individual, presencial e em Libras. $O$ pesquisador principal (estudante surdo) conduziu a pesquisa de opinião sozinho, apenas sanando algumas raras dúvidas com o intérprete. Vale comentar que o convite aos participantes ocorreu por conveniência, pois os voluntários estudavam na mesma instituição de ensino que o pesquisador principal. Por esse motivo, ocorreu a participação de um estudante de um curso de Pedagogia, que não era da área de Computação. A intenção era a de recrutar participantes surdos, usuários da Libras, que tivessem contato com a área de Computação, porém, considerando todas instituições de ensino superior da cidade, no período de realização da pesquisa (ano de 2017), os únicos dois estudantes surdos eram o pesquisador principal e um dos participantes voluntários. Houve algumas tentativas de realizar a pesquisa de opinião de forma remota, com estudantes surdos de outras localidades, porém, não obteve-se sucesso.

\section{Resultados}

Primeiramente, é importante destacar que o conteúdo do questionário foi o mesmo aplicado na Pesquisa de Opinião 1 e na Pesquisa de Opinião 2, o que mudou foi somente o formato como as perguntas estavam dispostas na tela do computador. Levando isso em consideração, as respostas ao questionário são passíveis de comparação. O questionário foi composto por 25 perguntas. A primeira pergunta para conhecer o nome completo, que não será transcrito aqui devido ao respeito ao anonimato dos participantes. As respostas para as questões de número 2 a 16 constam no Quadro 2, as de número 17 a 24 constam no Quadro 3, e as respostas à questão 25 constam na forma textual, as quais foram traduzidas por um intérprete. 
Quadro 2. Respostas às perguntas de 2 a 16 do questionário.

\begin{tabular}{|l|c|c|}
\hline Perguntas & Participante 1 & Participante 2 \\
\hline 2. Idade & 17 & Masculino \\
\hline 3. Sexo & Masculino & 9 \\
\hline $\begin{array}{l}\text { 4. Nível de conhecimento em Libras (auto } \\
\text { avaliação) }\end{array}$ & 8 & Ensino superior completo \\
\hline 5. Nível de escolaridade & Ensino superior incompleto & Ciência da Computação \\
\hline 6. Curso de graduação & Pedagogia & \\
\hline 7. Instituição de formação, cidade e estado & Unic (antiga Faculdade Anhanguera), Rondonópolis-MT \\
\hline $\begin{array}{l}\text { 8. Classificação sobre a percepção de } \\
\text { existência de sinais da Libras para } \\
\text { termos específicos da área }\end{array}$ & \multicolumn{2}{|c|}{ Não se aplica } \\
\hline Conhece algum sinal para se referir ao termo: & \multicolumn{2}{|c|}{} \\
\hline 9. Engenharia de software? & Sim (vídeo) & Sim (vídeo) \\
\hline 10. Processos? & Não & Sim (vídeo) \\
\hline 11. Modelo de processo/ Ciclo de vida? & Não & Sim (vídeo) \\
\hline 12. Modelos de Processos Prescritivos? & Não & Sim (vídeo) \\
\hline 13. Modelo Cascata? & Não & Não \\
\hline 14. Modelo Incremental? & Não & Não \\
\hline 15. Modelo Evolucionário? & Não & Não \\
\hline 16. Modelo Espiral? & \multicolumn{2}{|c|}{} \\
\hline
\end{tabular}

No Quadro 2, dentre as respostas afirmativas para as questões de 9 a 13, consta entre parênteses a palavra 'vídeo' para destacar que houve a filmagem do sinal informado pelo participante durante a entrevista.

Quadro 3. Respostas às perguntas de 17 a 24 do questionário.

\begin{tabular}{|l|c|c|c|}
\hline Voto para sinal proposto para: & Participante 1 & Participante 2 & Parecer \\
\hline 17. Engenharia de Software & 1 & 1 & 1 \\
\hline 18. Processos & 2 & 1 & 0 \\
\hline 19. Modelo de processo/ Ciclo de vida & 2 & 1 & 0 \\
\hline 20. Modelos de Processos Prescritivos & 1 & 1 & 1 \\
\hline 21. Modelo Cascata & 1 & 1 & 1 \\
\hline 22. Modelo Incremental & 3 & 3 & 1 \\
\hline 23. Modelo Evolucionário & 3 & 1 & 0 \\
\hline 24. Modelo Espiral & 3 & 3 & 1 \\
\hline
\end{tabular}

Fonte própria. Legenda (Parecer): Votos na mesma opção $=1$; Votos em diferentes opções $=0$.

Como pode ser visto no Quadro 3, 05 (cinco) dentre 08 (oito) termos foram votados por ambos os participantes. Um desses sinais é aquele que consta no Quadro $1 \mathrm{e}$ os demais constam ilustrados na Figura 2.

Figura 2. Quatro sinais votados por ambos os participantes.

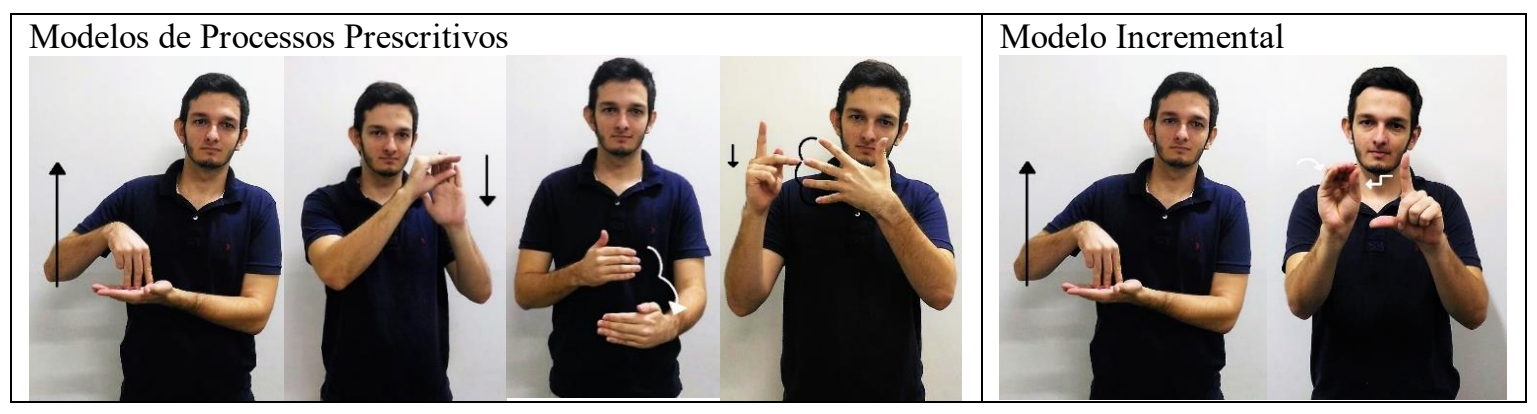




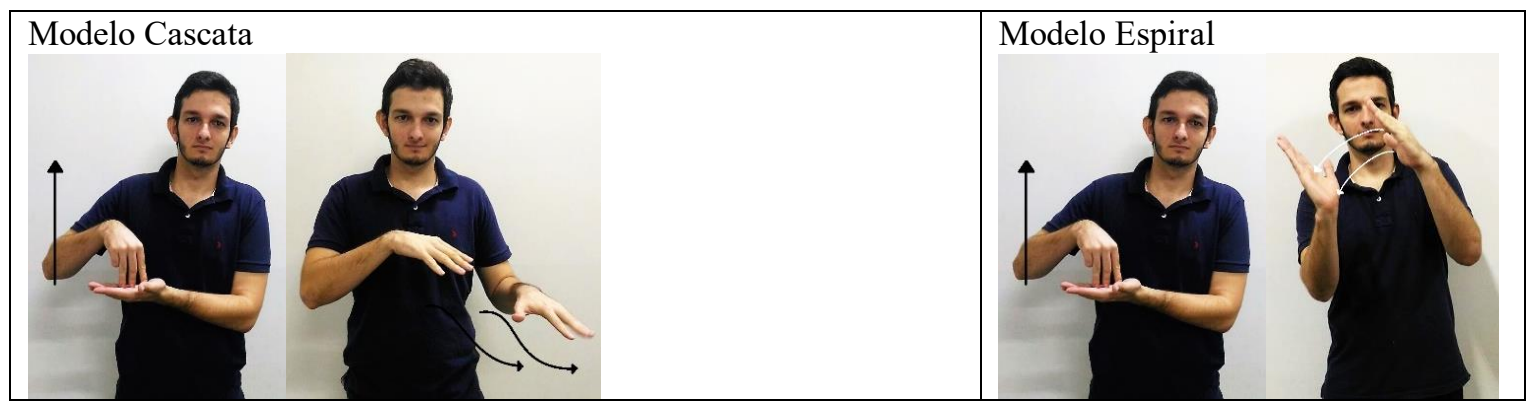

Fonte própria (Fotos do primeiro autor).

Com relação à pergunta 25 , se os entrevistados consideravam importante criar sinais da Libras para áreas de conhecimento que ainda possuem poucos sinais, como a computação, por exemplo. Ambos responderam que é importante.

\section{Considerações finais}

O objetivo geral era auxiliar no processo de ensino-aprendizagem, a partir da construção colaborativa de signos linguísticos em Libras para termos técnicos para a disciplina de Engenharia de Software presente em cursos da área de Computação. Transcorrido o desenvolvimento do trabalho, foi possível alcançar parcialmente o objetivo apresentado. A contribuição principal foi a colaboração entre o estudante surdo (autor principal desse artigo) com o professional intérprete da Libras na construção dos signos linguísticos para para termos selecionados. Essa construção contou com esforço, estudo e dedicação de ambos. O ponto que apresentou limitações foi a quantidade de participantes voluntários na pesquisa de opinião.

Além disso, também foi possível o desenvolvimento de novas termologias derivadas de conceitos pré-existentes fundamentados e suas definições conforme previsto no projeto. A elaboração de fichas catalogadas com cada termo-acadêmico-cientifica foi essencial no decorrer de cada entrevista o que proporcionou a aplicação prática dos conceitos assimilados. A realização de entrevistas se mostrou favorável à aceitação destes novos termos pelos participantes voluntários.

A intenção é mostrar que a construção de termos linguísticos significativos e suas termologias, contribuem muito a um conhecimento inteligível e sua assimilação. Além disso, pode ser um incentivo para que novos trabalhos acadêmicos, projetos e software voltados aos estudantes surdos possam ser produzidos, alcançando toda a comunidade surda. Portanto, esse trabalho poderá promover atração de mais estudantes surdos para cursos da área de computação, ampliar as condições para compreensão dos conteúdos por estudantes matriculados em cursos da área, colaborar com a ampliação de vocabulário para a Libras, e tentar padronizar sinais de jargão específico da engenharia de software, independente de regionalismos da língua.

Uma possibilidade de continuidade desta pesquisa é levar os signos linguísticos construídos para discussão no Fórum de Estudos dos Surdos na Área da Informática (FESAI), cujo objetivo "é desenvolver e incentivar pesquisas para criação de novos sinais e existentes de sinais de informática para divulgar na educação básica e no ensino 
superior" (FESAI, 2017). Os sinais são propostos e votados de forma colaborativa e democrática pelos participantes, com pessoas que atuam na área de informática.

\section{Referências}

Brasil. Lei $\mathrm{n}^{\mathrm{o}}$. 10.436, de 24 de abril de 2002. Dispõe sobre a Língua Brasileira de Sinais - Libras e dá outras providências.

Brasil. Decreto $\mathrm{n}^{\mathrm{0}} 5.626$, de 22 de dezembro de 2005. Regulamenta a Lei ${ }^{0} 10.436$, de 24 de abril de 2002, que dispõe sobre a Língua Brasileira de Sinais - Libras.

Borges, Lucas C.; Obara, Eduardo N.; Leite, Claudia Z.; Rocha, Fabiano G. (2015). Glossário interativo de Libras para a área de Computação. Computer on the Beach, Florianópolis/SC.

Boscarioli, Clodis; Galante, Guilherme; Oyamada, Marcio Seiji; Zara, Reginaldo A., Villwock, Rosangela. (2015). Aluno Surdo na Ciência da Computação: Discutindo os Desafios da Inclusão. XXIII Workshop sobre Educação em Informática, Recife, PE.

Brochado, Sonia Maria D.; Lacerda, Cristina Broglia de F.; Rocha, Luiz Renato M. da. (2016). Projeto de Pesquisa: Software Glossário de Informática com Aplicação de Libras e de Tecnologia de Captura de Movimento 3D. Journal of Research in Special Educational Needs, Volume 16, Number s1, p. 905-908.

Faqueti, Charlles Giovany; Grandi, Gilberto; Fantini, Liliane S.; Lorenzetti, Maria Lúcia. (2005). InfoLIBRAS - O Uso da Web para o Aprendizado da Língua de Sinais com Termos da Informática. XXV Congresso da Sociedade Brasileira de Computação, XXII Workshop de Informática na Escola (WIE), São Leopoldo/RS.

FESAI. Fórum de Estudos dos Surdos na Área da Informática. Disponível em: https://fesaiblog.wordpress.com/, acesso dia: 10/04/2017.

Kuhn, Talícia do Camo G. (2015). Processo de Criação de Termos Técnicos em Libras para Engenharia de Produção. Dissertação de Mestrado em Ensino de Ciência e Tecnologia, da Universidade Tecnológica Federal do Paraná. Ponta Grossa/PR.

Oliveira, Janine S.; Stumpf, Marianne R. (2013). Desenvolvimento de glossário de Sinais Acadêmicos em ambiente virtual de aprendizagem do curso Letras-Libras. INFORMÁTICA NA EDUCAÇÃO: teoria \& prática, Porto Alegre, v. 16, n. 2.

Paula Filho, Wilson de Pádua. (2015). Engenharia de Software: fundamentos, métodos e padrões. 3.ed. - [Reimpr.]. - Rio de Janeiro: LTC.

Pressman, Roger S. (2011). Engenharia de Software: Uma Abordagem Profissional. 7.ed. - Porto Alegre: AMGH.

Sommerville, Ian. Software Engineering. São Paulo: Pearson Addison Weslwy, 2003.

Souza, Celso Luiz de; Lima, Vera Lucia de S. e; Pádua, Flávio Luís Cardeal. Abordagem Interdisciplinar para a Criação e Preservação de Novos Sinais para Dicionários Terminológicos em Libras. Acta Semiótica et Lingvistica, Universidade Federal da Paraíba, v. 19, n. 1, 2014. Disponível em: goo.gl/hFb8Pe, acesso dia: 06/04/2017.

Wazlawick, Raul Sidnei. Engenharia de Software: conceitos e práticas. Rio de Janeiro: Elsevier, 2013. 